\title{
Stimulus induced behaviours in Tourette's syndrome
}

\author{
Valsamma Eapen, John Moriarty, Mary M Robertson
}

\begin{abstract}
Three cases of Gilles de la Tourette's syndrome (GTS) who experienced different tic related behaviours in response to both external and internal stimuli are described. Such behaviours might represent sensory tics, reflex motor tics, exaggerated startle responses, or obsessive compulsive behaviours. It is suggested that there is a continuum of stimulus induced behaviours, and that, in some cases, separations into individual descriptive entities may be difficult. There also seems to be a continuum between internal and external phenomena that induce the behaviours. Implications for the relation between GTS and obsessive compulsive disorder are also discussed.
\end{abstract}

(F Neurol Neurosurg Psychiatry 1994;57:853-855)

Despite renewed interest in the relation between Gilles de la Tourette's syndrome (GTS) and startle responses, ${ }^{1}$ the phenomenon of stimulus induced behaviours has not received much attention.

Sensory tics, a term coined by Shapiro et $a{ }^{2}{ }^{2}$ describes tics that are preceded by a sensation (itch, stretch, tightness, tingling, etc) in those parts of the body where the tics occur and the tic relieves the sensation. ${ }^{3-5}$

Tics may also occur in response to internal or external stimuli. Commander et $a l^{1}$ described two patients with GTS, with "reflex tics" in whom the tics were triggered by external stimuli (motor and vocal tics in response to other people coughing and sniffing). It may be argued, however, that these are not reflex tics but merely exaggerated startle responses. The relation between GTS and exaggerated startle responses has been noted by previous authors. ${ }^{6-8}$ Furthermore, tics may be complex and interwoven with obsessive compulsive behaviour. The obsessive compulsive behaviours in classic obsessive compulsive disorder are of course often preceded by a general sense of being ill at ease, which is relieved by the execution of the behaviours.

We describe three cases to illustrate a range of behaviours occurring in response to internal and external stimuli in patients with GTS.

\section{Case reports}

CASE 1

A single, 19 year old man living with his parents was referred to The National Hospital for Neurology and Neurosurgery, Queen Square, London. His first tic began at the age of seven with a neck twist, and since then he has had a wide repertoire of motor tics of the face, neck, head, shoulders, abdomen, and limbs. Vocalisations began at the age of 14, and have included grunting, throat clearing, gulping, sniffing, and clicking noises. $\mathrm{He}$ also has had a variety of obsessive compulsive behaviours such as arithmomania, a need to touch objects, checking behaviours (gas, doors, lights), a concern with symmetry, and being excessively tidy. He described a feeling in his chest like a stretch or tightness. ("A pain which doesn't hurt; I can't explain, like a hand inside squeezing.") This was followed by complex movements (for example, putting both feet together, jumping up and trying to kick his bottom to inflict pain), vocal tics (grunting, coughing, and shouting in a loud voice), and rituals (going upstairs, squeezing up into a ball, and hitting the bed or the wall). He performs these behaviours specifically to relieve the uncomfortable sensation or tension and thus this would fit with the current definition of a sensory tic. The sensation and its relief could, however, also be seen as compatible with the anxiety of obsessive compulsive disorder.

Family history of note is that his mother has a variety of motor and vocal tics and obsessive compulsive behaviours, his brother has motor and vocal tics, and his maternal grandmother and aunt are said to have obsessive compulsive behaviours. Both parents were interviewed and the tics were seen.

His birth and early development were normal. Mental state examination was normal except for the motor and vocal tics, and physical examination did not show any abnormality. Investigations including full blood count, acanthocytes, serum copper, ceruloplasmin and uric acid, EEG, and neuropsychological assessment were all within normal limits.

\section{CASE 2}

A 31 year old single, employed woman was referred to the National Hospital for Neurology and Neurosurgery by a local psychiatrist for evaluation. Her first tics were excessive blinking of the eyes at the age of 10 . 
Since then she has had a wide variety of motor tics of the face, shoulder, neck, and limbs. She admitted to vocalisations including sniffing, throat clearing, barking, and shrieking. She had coprolalia, which began at the age of 18. She has also had self injurious behaviour such as hitting herself, banging her leg, and pinching and squeezing herself. She also had many obsessive compulsive symptoms. Initially her obsessional thoughts revolved around newspapers, paperback books, and telephone directories. At the age of 26 , this was replaced by motor coaches. If she either saw a coach, heard the word "coach" or said the word "coach" herself, she felt anxious, fidgety, and possibly contaminated; so much so that she had tics (especially eye blinking), had to wash herself, and finally to change her clothes. She also has arithmomania with respect to coaches, in that if she sees a coach while driving, she closes her eyes and counts to 10 in an obsessional way 10 times. Having done this, when she gets home, she washes and changes her clothes. This was very distressing for her as well as being socially disabling. Typically, the symptoms were made worse by tiredness and anxiety. She was able to suppress the symptoms for brief periods at the expense of mounting inner tension. This patient's symptoms could be obsessive compulsive disorder anxiety but are also a clear description of reflex tics.

Her birth and early childhood were unremarkable. She did well at school and work. Her father was reported to be obsessional and was a compulsive cleaner. Her mother developed anxiety and depression at the age of 40 and was treated with benzodiazepines.

Neurological and mental state examination were normal apart from the motor tics and vocalisations. Investigations including EEG, serum copper, ceruloplasmin and uric acid, full blood count, and acanthocytes were within normal limits. She was successfully treated with a combination of fluvoxamine and haloperidol.

CASE 3

A man presented to the National Hospital for Neurology and Neurosurgery at the age of 28 . His symptoms began at the age of seven with obsessional rituals. If he touched a person he had to rub something else. Since then he has had various obsessional actions including folding rituals and turning knobs on doors. His first motor tics began at the age of 19 and have included a wide variety of facial, neck, head, abdomen, and limb tics. He also had vocalisations including grunting, throat clearing, coughing, clicking, and panting. Also, he would utter a whole obscene statement accompanied by motor and vocal tics, precipitated by hearing somebody else coughing (if someone coughs, he puts his hand over his ears, bends his knees, and propels himself with an expulsion of air accompanied by a noise resembling a cough). He also developed motor tics if he saw somebody else spitting in the street. These were not a form of echopraxia, in that he developed a variety of motor tics and was not copying the spitting behaviour. He also had obsessive compulsive rituals in that he would count the number of times someone coughed, so that he could go home and perform a ritual (for example, folding his clothes) the same number of times. $\mathrm{He}$ also had self injurious behaviour and felt forced to touch objects, but exhibited no copropraxia or echophenomena. The symptoms increased with stress and had a waxing and waning course.

He had had a normal birth and his childhood was unremarkable. The only family history of note was that his mother had had a puerperal depression for which she was treated with electroconvulsive therapy. Neurological and mental state examinations were normal apart from the motor and vocal tics. An EEG was normal, as were serum copper and ceruloplasmin, full blood count, acanthocytes, and blood chemistry.

\section{Discussion}

We have presented three cases of GTS in which tics and obsessive compulsive behaviour occurred in response to internal (tightness in the chest) and external (other people coughing, spitting, and seeing or hearing the word "coaches") stimuli. In all our cases, these phenomena were interwoven and occurred in response to evocative stimuli. In the first case the main response was that of complex motor tic, although accompanied by compulsive rituals. In the second case the compulsive rituals with a clear cognitive component were predominant, whereas in the third case both motor tics and compulsive phenomena were equally prominent. Although there is considerable overlap between these stimulus induced tics and obsessive compulsive behaviours, there are cogent arguments for avoiding describing them with uniform terminology. In particular because the pharmacological treatments of tics and obsessive compulsive behaviour are different.

Obsessive compulsive behaviour is now recognised as an integral part of GTS. ${ }^{910}$ Robertson et al ${ }^{11}$ documented that obsessive compulsive behaviour was significantly associated with features of GTS such as coprophenomena and echophenomena. Our family studies have also suggested a genetic relation between obsessive compulsive behaviour and GTS. ${ }^{12-14}$

Another term that may be used in the descriptive phenomenology of these conditions is "impulsions". Shapiro et $a l^{2}$ first described an "impulsion" (as defined by Hinsie and Campbell ${ }^{15}$ ) in GTS as a stimulus that sets the mind in action. The stimulus may originate in the objective world, in the subject himself, in his soma, or in his psyche, including its conscious or its unconscious parts. By implication, this wide definition includes tics as well as obsessive compulsive phenomena. Impulsions could be either reflex or sensory tics. Another phenomenon, "mental play" 
(playful impulsions), has also been described in GTS by Cath et al. ${ }^{16}$ They suggest that patients find these experiences enjoyable and often do not try to resist them. Therefore they argue that this phenomenon is distinct from obsessive compulsive disorder. Echophenomena, seen in some $11 \%$ to $44 \%$ of patients with GTS $^{8}$ is also within the continuum of stimulus induced behaviours.

It is clear from our case descriptions that these patients with GTS have both simple sensory phenomena and more complex cognitive experiences. It could be argued that the first, with their involuntary motor and vocal responses, are more related to the tic end of the continuum, and the second to the obsessive compulsive end. But this must at least be a rather arbitrary divide. There is also debate as to whether these behaviours are voluntary or involuntary. Obeso et $a l^{17}$ found that the normal premovement (Bereitschafts) potential associated with voluntary initiated motor acts was not evident before spontaneous simple tics in patients with GTS. It is highly unlikely that the more elaborate ritual would not have the electrophysiological appearances of voluntary movements, although this has not been tested.

George et $a l^{18}$ reported that the compulsions in GTS arose spontaneously, whereas they were often preceded by cognitions in primary obsessive compulsive disorder. In some patients these phenomena may form part of the obsessive compulsive disorder/impulsion end of the continuum in GTS, and are triggered by an internal stimuli. From this argument, it may well be that in others, these behaviours occur in response to external stimuli and fall at the tic end of the continuum. The authors suggest that the term reflex tic would be best suited for those tics that occurred in response to an external stimulus (for example, someone else coughing) and the term sensory tic be reserved for tics occurring in response to stimuli arising in the soma (for example, a tingling sensation) or an internal stimulus (for example, oneself coughing).

Whereas it is attractive to try and make distinctions between reflex tics, sensory tics, and impulsions, we believe that the overlap between these behaviours makes such distinctions extremely difficult. It seems that the stimuli that induce tics and obsessive compulsive behaviour form a continuum between internal and external phenomena and the behaviours performed in response to these stimuli form a continuum between tics and obsessive compulsive behaviour. These responses may or may not share a common pathogenesis, and may respond differentially to treatment. We argue that, whereas we can strive to describe these phenomena as accurately as possible, we are not in a position to be dogmatic about this area of phenomenology. Treatment may often be empirical and until the pathogenesis of these behaviours is more clearly understood, we should not impose descriptive categories that fail to describe the full range of clinical manifestations.

1 Commander M, Corbett J, Prendergast M, Ridley C. Reflex tics in two patients with Gilles de la Tourette Reflex tics in two patients with Gilles de

2 Shapiro AK, Shapiro ES, Young JG, Feinberg TE. Shapiro AK, Shapiro ES, Young JG, Feinberg TE.
Sensory tics. In: Shapiro AK, Shapiro ES, Young JG, Sensory tics. In: Shapiro AK, Shapiro ES, Young JG,
Feinberg TE, eds. Gilles de la Tourette Syndrome. 2nd ed. Feinberg TE, eds. Gilles de la Tourette Sym

3 Bliss J. Sensory experiences of Gilles de la Tourette Syndrome. Arch Gen Psychiatry 1980;37:1343-7.

4 Bullen JG, Hemsley R. Sensory experience as a trigger in Gilles de la Tourette Syndrome. F Behav Ther Exp Psychiatry 1983;14:197-201.

5 Kurlan R, Lichter D, Hewitt D. Sensory tics in Tourette's syndrome. Neurology 1989;39:731-4.

6 Corbett JA. Tics and Tourette's syndrome. In: Rutter M, Hirsov L, eds. Child psychiatry: modern approaches. Philadelphia: Lippincott, 1976:674-87.

7 Lees AJ, Robertson M, Trimble MR, Murray NMF. A clinical study of Gilles de la Tourette Syndrome in the United Kingdom. $f$ Neurol Neurosurg Psychiatry 1984;47:1-8.

8 Robertson MM. The Gilles de la Tourette syndrome: the current status. $\operatorname{Br} \mathcal{F}$ Psychiatry 1989;154:147-69.

9 Frankel M, Cummings JL, Robertson MM, Trimble MR, Hill MA, Benson DF. Obsessions and Compulsions in Gilles de la Tourette Syndrome. Neurology 1986;36: 378-83.

10 Pauls DL, Towbin KE, Leckman JF, Zahner GEP, Cohen DJ. Gilles de la Tourette syndrome and obsessive-compulsive disorder. Arch Gen Psychiatry 1986;43:1180-2.

11 Robertson MM, Trimble MR, Lees AJ. The psychopathology of the Gilles de la Tourette syndrome: a phenomenological analysis. Br $\mathcal{f}$ Psychiatry 1988;152: 383-90.

12 Robertson MM, Gourdie A. Familial Tourette's syndrome in a large British pedigree: associated psy-
chopathology, severity of Tourette's and potential for chopathology, severity of Tourette's and potent

13 Curtis D, Robertson MM, Gurling HMD. Autosomal dominant gene transmission in a large kindred with Gilles de la tourette syndrome. Br f Psychiatry 1992; Gilles de la

14 Eapen V, Pauls DL, Robertson MM. Autosomal dominant gene transmission in Gilles de la Tourette syndrome: United Kingdom cohort study. $\mathrm{Br} F$ Psychiatry 1994 (in press).

15 Hinsie LE, Campbell RJ. Psychiatric Dictionary. 4th ed. New York: Oxford University Press, 1970

16 Cath DC, van de Wetering BJM, van Woerkom TCAM, Hoogduin CAL, Roos RAC, Rooijmans HGM. Mental play in Gilles de la Tourette syndrome and obsessive play in Gilles de la Touretse syndrome and obsess.

17 Obeso JA, Rothwell JC, Marsen CD. The neurophysiology of Tourette syndrome. In: Friedhoff AJ, Chase TN, eds. Gilles de la Tourette syndrome: Advances in Neurology. eds. Gilles de la Tourette syndrome: Advance
Vol 35. New York: Raven press, 1982 .

18 George MS, Trimble MR, Ring HA, Sallee FR, Robertson MM. Obsessions in obsessive compulsive Robertson MM. Obsessions in obsessive compulsive
disorder with and without Gilles de la Tourette's syndisorder with and without Gilles de la
drome. Am $\mathcal{F}$ Psychiatry 1993;150:93-7. 